\title{
Calculation of the Hadronic Vacuum Polarization Disconnected Contribution to the Muon Anomalous Magnetic Moment
}

\author{
T. Blum, ${ }^{1}$ P. A. Boyle, ${ }^{2}$ T. Izubuchi, ${ }^{3,4}$ L. Jin, ${ }^{5}$ A. Jüttner, ${ }^{6}$ C. Lehner, ${ }^{3,}$ K. Maltman, ${ }^{7,8}$ M. Marinkovic, \\ A. Portelli, ${ }^{2,6}$ and M. Spraggs ${ }^{6}$ \\ (RBC and UKQCD Collaborations) \\ ${ }^{1}$ Physics Department, University of Connecticut, Storrs, Connecticut 06269-3046, USA \\ ${ }^{2}$ SUPA, School of Physics, The University of Edinburgh, Edinburgh EH9 3JZ, United Kingdom \\ ${ }^{3}$ Physics Department, Brookhaven National Laboratory, Upton, New York 11973, USA \\ ${ }^{4}$ RIKEN-BNL Research Center, Brookhaven National Laboratory, Upton, New York 11973, USA \\ ${ }^{5}$ Physics Department, Columbia University, New York, New York 10027, USA \\ ${ }^{6}$ School of Physics and Astronomy, University of Southampton, Southampton SO17 1BJ, United Kingdom \\ ${ }^{7}$ Mathematics \& Statistics, York University, Toronto, Ontario M3J 1P3, Canada \\ ${ }^{8}$ CSSM, University of Adelaide, Adelaide, South Australia 5005, Australia \\ ${ }^{9}$ CERN, Physics Department, 1211 Geneva 23, Switzerland
}

(Received 30 December 2015; revised manuscript received 6 April 2016; published 8 June 2016)

\begin{abstract}
We report the first lattice QCD calculation of the hadronic vacuum polarization (HVP) disconnected contribution to the muon anomalous magnetic moment at physical pion mass. The calculation uses a refined noise-reduction technique that enables the control of statistical uncertainties at the desired level with modest computational effort. Measurements were performed on the $48^{3} \times 96$ physical-pion-mass lattice generated by the RBC and UKQCD Collaborations. We find the leading-order hadronic vacuum polarization $a_{\mu}^{\mathrm{HVP}(\mathrm{LO}) \text { disc }}=-9.6(3.3)(2.3) \times 10^{-10}$, where the first error is statistical and the second systematic.
\end{abstract}

DOI: 10.1103/PhysRevLett.116.232002

Introduction.-The anomalous magnetic moment of leptons provides a powerful tool to test relativistic quantum-mechanical effects at tremendous precision. Consider the magnetic dipole moment of a fermion

$$
\vec{\mu}=g\left(\frac{e}{2 m}\right) \vec{s}
$$

where $\vec{s}$ is the particle's spin, $e$ is its charge, and $m$ is its mass. While Dirac's relativistic quantum-mechanical treatment of a fermion coupled minimally to a classical photon background predicts a Landé factor of $g=2$, additional electromagnetic quantum effects allow the anomalous magnetic moment $a=(g-2) / 2$ to assume a nonzero value. These anomalous moments are measured very precisely. For the electron, e.g., $a_{e}=$ $0.00115965218073(28)$ [1] yielding the currently most precise determination of the fine structure constant $\alpha=1 / 137.035999157(33)$ based on a five-loop quantum electrodynamics (QED) computation [2].

Published by the American Physical Society under the terms of the Creative Commons Attribution 3.0 License. Further distribution of this work must maintain attribution to the author(s) and the published article's title, journal citation, and DOI.
The muon anomalous magnetic moment promises high sensitivity to new physics (NP) beyond the standard model $(\mathrm{SM})$ of particle physics. In general, new physics contributions to $a_{\ell}$ are expected to scale as $a_{\ell}-a_{\ell}^{\mathrm{SM}} \propto$ $\left(m_{\ell}^{2} / \Lambda_{\mathrm{NP}}^{2}\right)$ for lepton $\ell=e, \mu, \tau$ and new physics scale $\Lambda_{\mathrm{NP}}$. With $a_{\tau}$ being currently experimentally inaccessible, $a_{\mu}$ is the optimum channel to uncover new physics.

Interestingly, there is a $3.1 \sigma-3.5 \sigma$ tension between current experimental and theoretical determinations of $a_{\mu}$,

$$
\begin{aligned}
a_{\mu}^{\text {expt }}-a_{\mu}^{\mathrm{SM}}= & (27.6 \pm 8.0) \times 10^{-10}[3], \\
& (25.0 \pm 8.0) \times 10^{-10}[4],
\end{aligned}
$$

where the experimental measurement is dominated by the BNL experiment E821 [3]. The theoretical prediction [4] is broken down in individual contributions in Table I.

The theory error is dominated by the hadronic vacuum polarization (HVP) and hadronic light-by-light (HLBL) contributions. A careful first-principles determination of these hadronic contributions is very much desired to resolve or more firmly establish the tension with the current SM prediction. Furthermore, the future $a_{\mu}$ experiments at Fermilab (E989) [9] and J-PARC (E34) [10] intend to decrease the experimental error by a factor of 4 . Therefore, 
TABLE I. Current standard model prediction of $a_{\mu}$ including uncertainties contrasted with the experimental target precision of the upcoming Fermilab E989 experiment [4-6]. The individual contributions are defined in Ref. [4].

\begin{tabular}{|c|c|c|}
\hline Contribution & Value $\times 10^{10}$ & $\begin{array}{r}\text { Uncertainty } \\
\times 10^{10}\end{array}$ \\
\hline QED & 11658471.895 & 0.008 \\
\hline Electroweak corrections & 15.4 & 0.1 \\
\hline HVP [leading order (LO)] [7] & 692.3 & 4.2 \\
\hline $\operatorname{HVP}(\mathrm{LO})[8]$ & 694.9 & 4.3 \\
\hline HVP (next-to-leading order) & -9.84 & 0.06 \\
\hline $\begin{array}{l}\text { HVP (next-to-next-to-leading } \\
\text { order) }\end{array}$ & 1.24 & 0.01 \\
\hline HLBL & 10.5 & 2.6 \\
\hline Total SM prediction [7] & 11659181.5 & 4.9 \\
\hline Total SM prediction [8] & 11659184.1 & 5.0 \\
\hline BNL E821 result & 11659209.1 & 6.3 \\
\hline Fermilab E989 target & & $\approx 1.6$ \\
\hline
\end{tabular}

a similar reduction of the theory error is essential in order to make full use of the experimental efforts.

The current SM prediction for the HLBL contribution [11] is based on a model of quantum chromodynamics (QCD); however, important progress towards a first-principles computation has been made recently [12-14]. The uncertainty of the HVP contribution may be reduced to $\delta a_{\mu}=2.6 \times 10^{-10}$ using improved experimental $e^{+} e^{-}$ scattering data [5]. An ab initio theory prediction based on QCD, however, can provide an important alternative determination that is systematically improvable to higher precision.

One of the main challenges in the first-principles computation of the HVP contribution with percent or subpercent uncertainties is the control of statistical noise for the quark-disconnected contribution (see Fig. 1) at physical pion mass. Significant progress has been made recently in the computation of an upper bound [15-17], an estimate using lattice QCD data at heavy pion mass [18], and towards a first-principles computation at physical pion mass [19]. Here, we present the first result for
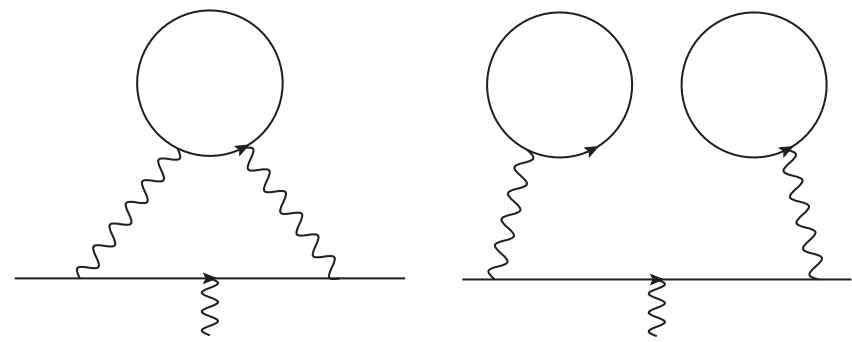

FIG. 1. HVP contributions to $a_{\mu}$ with the external photon attached to the muon line. As is common for nonperturbative lattice QCD computations, one does not explicitly draw gluons but understands each diagram to stand for all orders in QCD.
$a_{\mu}^{\mathrm{HVP}(\mathrm{LO}) \text { disc }}$ at physical pion mass. We report the result for the combined up, down, and strange-quark contributions.

Computational method.-In the following we describe the refined noise-reduction technique that allowed for the control of the statistical noise with modest computational effort.

We follow the basic steps of Ref. [20] and treat the muon and photon parts of the diagrams in Fig. 1 analytically, writing

$$
a_{\mu}=4 \alpha^{2} \int_{0}^{\infty} d\left(q^{2}\right) f\left(q^{2}\right)\left[\Pi\left(q^{2}\right)-\Pi\left(q^{2}=0\right)\right],
$$

where $f\left(q^{2}\right)$ is a known analytic function [20] and $\Pi\left(q^{2}\right)$ is defined in the continuum through the two-point function

$$
\sum_{x} e^{i q x}\left\langle J_{\mu}(x) J_{\nu}(0)\right\rangle=\left(\delta_{\mu \nu} q^{2}-q_{\mu} q_{\nu}\right) \Pi\left(q^{2}\right),
$$

with the sum over space-time coordinate $x$ and $J_{\mu}(x)=i \sum_{f} Q_{f} \bar{\Psi}_{f}(x) \gamma_{\mu} \Psi_{f}(x)$. The sum is over quark flavors $f$ with QED charge $Q_{f} \quad\left(Q_{u}=2 / 3\right.$, $\left.Q_{d / s}=-1 / 3\right)$. We compute $\Pi\left(q^{2}\right)$ using the kernel function of Refs. [21,22],

$\Pi\left(q^{2}\right)-\Pi\left(q^{2}=0\right)=\sum_{t}\left(\frac{\cos (q t)-1}{q^{2}}+\frac{1}{2} t^{2}\right) C_{\text {all }}(t)$,

with

$$
C_{\text {all }}(t)=\frac{1}{3} \sum_{\vec{x}} \sum_{j=0,1,2}\left\langle J_{j}(\vec{x}, t) J_{j}(0)\right\rangle,
$$

which sufficiently suppresses the short-distance contributions such that we are able to use two less computationally costly, nonconserved, local lattice vector currents [23]. For convenience, we have split the space-time sum over $x$ in a spatial sum over $\vec{x}$ and a sum over the time coordinate $t$. We sum over spatial Lorentz indices $0,1,2$.

The Wick contraction in Eq. (6) yields both connected and disconnected diagrams of Fig. 1. In the following $C$ stands for the combined up, down, and strange-quark disconnected contribution of $C_{\text {all }}$, while $C_{s}$ stands for the strange-quark connected contribution of $C_{\text {all }}$. The reason for defining $C_{s}$ becomes apparent below. The light up and down flavors are treated as mass degenerate such that

$$
C(t)=\frac{1}{3 V} \sum_{j=0,1,2} \sum_{t^{\prime}}\left\langle\mathcal{V}_{j}\left(t+t^{\prime}\right) \mathcal{V}_{j}\left(t^{\prime}\right)\right\rangle_{\mathrm{SU}(3)},
$$

where $V$ stands for the four-dimensional lattice volume, $\mathcal{V}_{\mu}=(1 / 3)\left(\mathcal{V}_{\mu}^{u / d}-\mathcal{V}_{\mu}^{s}\right)$, the average is over all $\mathrm{SU}(3)$ gauge configurations, and 


$$
\mathcal{V}_{\mu}^{f}(t)=\sum_{\vec{x}} \operatorname{Im} \operatorname{Tr}\left[D_{\vec{x}, t ; \vec{x}, t}^{-1}\left(m_{f}\right) \gamma_{\mu}\right],
$$

with Dirac operator $D\left(m_{f}\right)$ evaluated at quark mass $m_{f}$.

Controlling statistical fluctuations is the largest challenge in the computation of the disconnected contribution. In order to successfully measure the disconnected contribution, two conditions need to be satisfied: (i) large fluctuations of the up, down, and strange contributions that enter with opposite sign need to cancel [15] and (ii) the measurement needs to average over the entire space-time volume without introducing additional noise. Here, we use the following method to satisfy both (i) and (ii) simultaneously. First, the full quark propagator is separated in high and low-mode contributions, where the former are estimated stochastically and the latter are averaged explicitly [24], i.e., we separate $D^{-1}=\sum_{n} v^{n}\left(w^{n}\right)^{\dagger}+D_{\text {high }}^{-1}$, where the vectors $v^{n}$ and $w^{n}$ are reconstructed from the even-odd preconditioned low modes $n$ of the Dirac propagator $D^{-1}$. It is now crucial to include all modes with eigenvalues up to the strange-quark mass in the set of low modes for the up and down-quark propagators to satisfy (i). Since the signal is the difference of light and strange contributions, we may then expect the high-mode contribution to be significantly suppressed and the low-mode contribution to contain the dominant part of the signal. This is indeed the case in our computation and yields a substantial statistical benefit since we evaluate the low modes exactly without the introduction of noise and average explicitly over the entire volume.

In order to satisfy (ii), we must control the stochastic noise of the high-mode contributions originating from unwanted long-distance contributions of the random $Z_{2}$ sources of Ref. [24]. We achieve this by using what we refer to as sparsened $Z_{2}$ noise sources that have support only for points $x_{\mu}$ with $\left[x_{\mu}-x_{\mu}^{(0)}\right] \bmod N=0$ thereby defining a sparse grid with spacing $N$, similar to Ref. [25]. While a straightforward dilution strategy [24] would require us to sum over all possible offsets of the sparse noise grid, $x_{\mu}^{(0)}$, we choose the offset stochastically for each individual source, which allows us to project to all momenta. It also allows us to avoid the largest contribution of such random sources to the noise that comes from random sources at nearby points.

The parameter choice of $N$ is crucial to satisfy (ii) with minimal cost. An interesting measure for the noise originating from the random sources is the variance $\sigma^{2}$ of the set of all numbers $\mathcal{V}_{\mu}(t)$ for all possible $\mu$ and $t$ sampled on a fixed gauge configuration; see Fig. 2. Since we can use all possible $O\left(M^{2}\right)$ combinations of $M$ high-mode sources and time coordinates in Eq. (7), we may expect a noise suppression of $O(1 / M)$ as long as individual contributions are sufficiently statistically independent. A similar idea of $O(1 / M)$ noise reduction was recently successfully used in Ref. [12]. We find this to hold to a large degree, and therefore also show the appropriately rescaled $\sigma$ in the

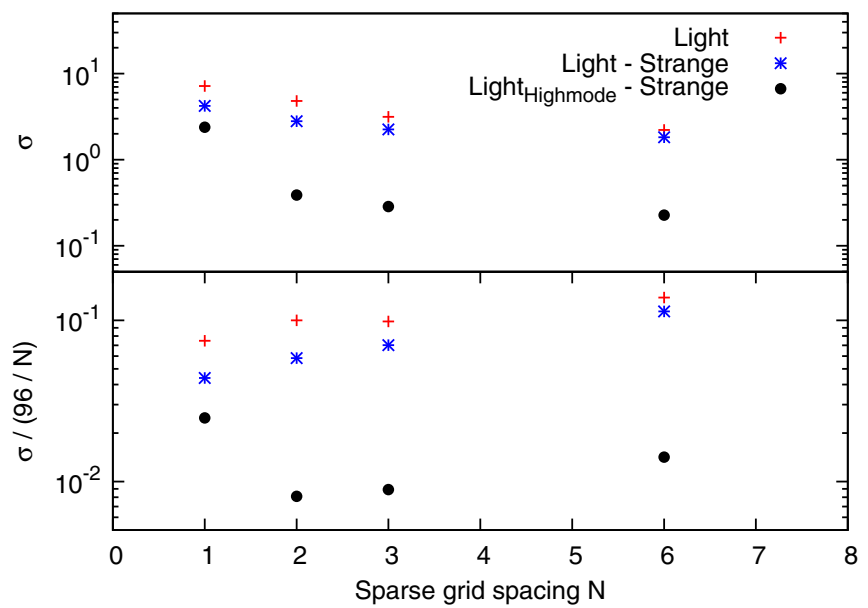

FIG. 2. Noise of the single vector operator loop as a function of sparse grid spacing $N$. The figure at the bottom normalizes the noise by taking into account the additional volume averaging for smaller values of $N$.

lower panel of Fig. 2. The figure illustrates the powerful cancellation of noise between the light and strange-quark contributions and the success of the sparsening strategy. We find an optimum value of $N=3$ for the case at hand, which is used for the subsequent numerical discussion.

We use 45 stochastic high modes per configuration and measure on the 21 Möbius domain wall [26] configurations of the $48^{3} \times 96$ ensemble at physical pion mass and lattice cutoff $a^{-1}=1.73 \mathrm{GeV}$ generated by the RBC and UKQCD collaborations [27]. For this number of high modes we find the QCD gauge noise to dominate the uncertainty for $a_{\mu}^{\mathrm{HVP}(\mathrm{LO}) \text { disc }}$. The all-mode-averaging strategy $[28,29]$ was employed to reduce the cost of computing multiple sources on the same configuration. The computation presented in this manuscript uses 2000 zMöbius [30] eigenvectors generated as part of an ongoing HLBL lattice computation [12]. We treat the shorter directions with 48 points as the time direction and average over the three symmetric combinations to further reduce stochastic noise. Additional details of our analysis are given in Ref. [31].

Analysis and results.-Combining Eqs. (3) and (5) and using $C(t)=C(-t)$,

$$
a_{\mu}^{\mathrm{HVP}(\mathrm{LO}) \mathrm{disc}}=\sum_{t=0}^{\infty} w_{t} C(t),
$$

with appropriately defined $w_{t}$. Because of our choice of relatively short time direction with 48 points, special care needs to be taken to control potentially missing long-time contributions in $C(t)$. In the following we estimate these effects quantitatively. Consider the vector operator $V_{\mu}^{f, f^{\prime}}(x)=\bar{\Psi}_{f}(x) \gamma_{\mu} \Psi_{f^{\prime}}(x)$ with $f$ and $f^{\prime}$ denoting quark flavors. Then the Wick contractions $\left\langle V_{\mu}^{u, u} V_{\nu}^{u, u}\right\rangle-$ $\left\langle V_{\mu}^{u, d} V_{\nu}^{d, u}\right\rangle$ isolate the light-quark disconnected contribution 
in the isospin symmetric limit; see also Ref. [32]. Unfortunately, there is no similar linear combination (without partial quenching) that allows for the isolation of the strange-quark disconnected contribution. Nevertheless, using $\left\langle\left(V_{\mu}^{u, u}-V_{\mu}^{s, s}\right)\left(V_{\nu}^{u, u}-V_{\nu}^{s, s}\right)\right\rangle-\left\langle V_{\mu}^{u, d} V_{\nu}^{d, u}\right\rangle$ one can isolate the sum of $C(t)+C_{s}(t)$, again making use of the isospin symmetry. Since this sum corresponds to a complete set of Feynman diagrams resulting from the above Wick contractions, we can represent it as a sum over individual exponentials $C(t)+C_{s}(t)=\sum_{m} c_{m} e^{-E_{m} t}$ with $c_{m} \in \mathbb{R}$ and $E_{m} \in \mathbb{R}^{+}$. The coefficients $c_{m}$ can be negative because positivity arguments only apply to some individual Wick contractions but not necessarily to the sum.

We show $C(t)$ and $C_{s}(t)$ obtained in our lattice QCD computation in Fig. 3. Starting from time slices 17 and 18 the correlator $C(t)$ is not well resolved from 0 ; however, from time slices 11 to 17 a two-state fit including the $\rho(770)$ and $\phi(1020)$ describes $C(t)+C_{s}(t)$ well. Here, the $\rho$ is a proxy for combined $\rho$ and $\omega$ contributions due to their similar energy. It is necessary to include the $\phi$ in this fit ansatz, in spite of it having a larger mass than the lower lying $\rho$ and $\omega$ states, because the combination of the octet vector current $I=0$ and $I=1$ polarizations representing $C(t)+C_{s}(t)$ in the isospin limit has relative coefficients that produce a strong $\rho-\omega$ cancellation. In the narrow width approximation, with physical values of the vector meson decay constants, this cancellation leads to an expectation $c_{\rho} / c_{\phi} \simeq-0.1$. This expectation is well borne out by the results of our fits. The vector meson states are not stable in our lattice simulation; however, the twoexponential fit form employed serves as a physically motivated model, albeit one that fits the data well. Since this model only enters our systematic error estimate, we find this imperfection to be acceptable. A systematic study of different fit ranges is presented in Fig. 4, where $p$ values greater than 0.05 are found for all fit ranges $t \in$ $\left[t_{\min }, \ldots, 17\right]$ with $t_{\min } \in[8, \ldots, 12]$.

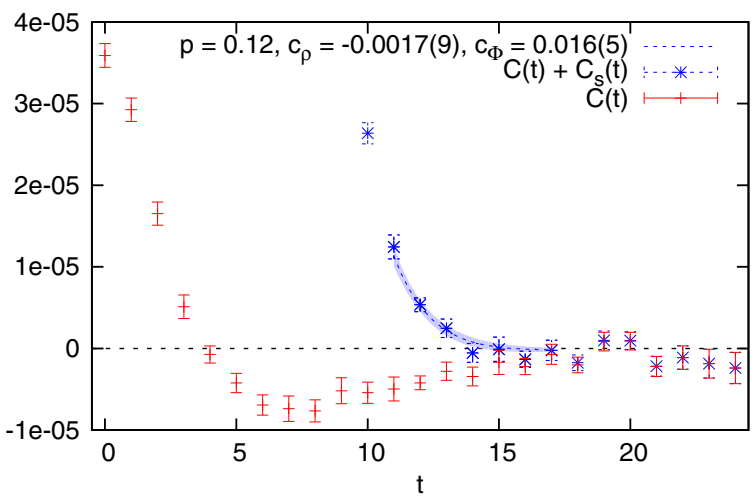

FIG. 3. Zero-momentum projected correlator $C(t)$ and $C(t)+C_{s}(t)$. A correlated fit of $\rho(770)$ and $\phi(1020)$ exponentials via $c_{\rho} e^{-E_{\rho} t}+c_{\phi} e^{-E_{\phi} t}$ in the region $t \in[11, \ldots, 17]$ to $C(t)+$ $C_{s}(t)$ yields a $p$ value of 0.12 . We use fixed energies $E_{\rho}=$ $770 \mathrm{MeV}$ and $E_{\phi}=1020 \mathrm{MeV}$ and fit parameters $c_{\rho}$ and $c_{\phi}$.
We now define the partial sums

$$
\begin{gathered}
L_{T}=\sum_{t=0}^{T} w_{t} C(t), \\
F_{T}(r)=\sum_{t=T+1}^{t_{\max }} w_{t}\left[c_{\rho}^{r} e^{-E_{\rho} t}+c_{\phi}^{r} e^{-E_{\phi} t}-C_{s}(t)\right],
\end{gathered}
$$

where $c_{\rho}^{r}$ and $c_{\phi}^{r}$ are the parameters of the fit with fit range $r$ and $t_{\max }=24$ for our setup. For sufficiently large $T, L_{T}$ is expected to exhibit a plateau region as a function of $T$ from which we can determine $a_{\mu}^{\mathrm{HVP}(\mathrm{LO}) \text { disc }}$. The sum $L_{T}+F_{T}$ is also expected to exhibit such a plateau to the extent that the model in $F_{T}$ describes the data well.

Based on Fig. 4, we choose $r=[11, \ldots, 17]$ as the preferred fit range to determine $F_{T}$ but a cross-check with $r=[12, \ldots, 17]$ has been performed yielding a consistent result. Figure 5 shows the resulting plateau region for $L_{T}$ and $L_{T}+F_{T}$. The substantial statistical noise in the large- $T$ region of Fig. 5 is a consequence of $w_{t}$ growing approximately with the fourth power of $t$ and the statistical noise of $C(t)$ being $t$ independent. In order to avoid contamination of our first-principles computation with the model dependence of $F_{T}$, we determine $a_{\mu}^{\mathrm{HVP}(\mathrm{LO}) \text { disc }}$ from $L_{T=20}$ and include $F_{T=20}$ as systematic uncertainty estimating a potentially missing long-time tail. We choose the value at $T=20$ since it appears to be safely within a plateau region but sufficiently far from $T=24$ to suppress backwardspropagating effects [33]. We find $a_{\mu}^{\mathrm{HVP}(\mathrm{LO}) \text { disc }}=$ $-9.6(3.3) \times 10^{-10}$.

We expect the finite lattice spacing and finite simulation volume as well as long-time contributions to Eq. (9) to dominate the systematic uncertainties of our result. With respect to the finite lattice spacing a reasonable proxy for the current computation may be our HVP connected strange-quark analysis [34] for which the $48^{3}$ result at $a^{-1}=1.73 \mathrm{GeV}$ agrees within $O(5 \%)$ with the continuumextrapolated value. The estimate of 5\% discretization errors for the current calculation is also of the expected size of

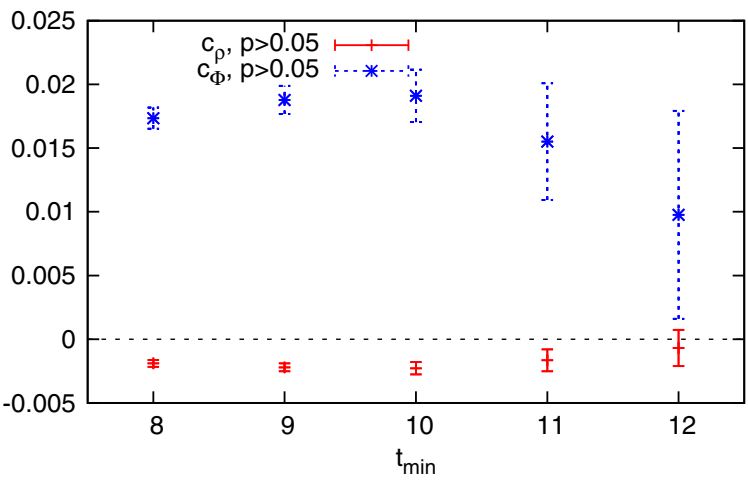

FIG. 4. Coefficients and $p$ values of a fit of $c_{\rho} e^{-E_{\rho} t}+c_{\phi} e^{-E_{\phi} t}$ to $C(t)+C_{s}(t)$ in the region $t \in\left[t_{\min }, \ldots, 17\right]$. 

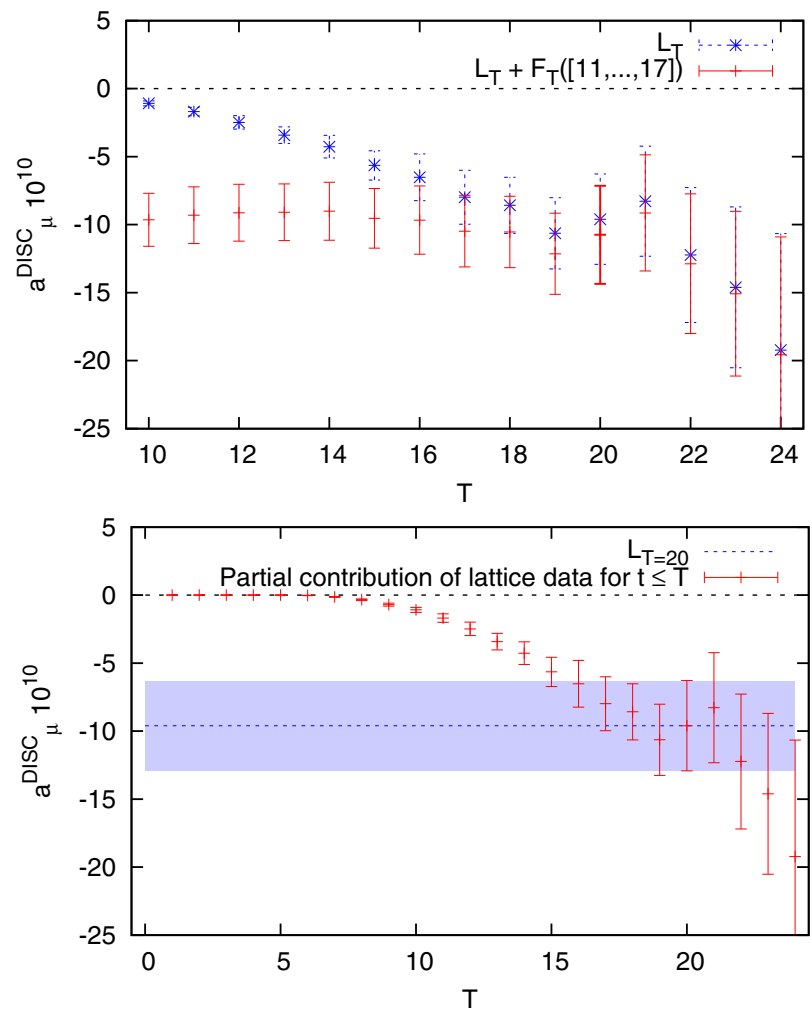

FIG. 5. The sum of $L_{T}$ and $F_{T}$ defined in Eqs. (10) and (11) has a plateau from which we read off $a_{\mu}^{\mathrm{HVP} \text { (LO) disc }}$. The lower panel compares the partial sums $L_{T}$ for all values of $T$ with our final result for $a_{\mu}^{\mathrm{HVP}(\mathrm{LO}) \text { disc }}$ with its statistical error band.

$O\left(a^{2} \Lambda_{\mathrm{QCD}}^{2}\right)$ with $\Lambda_{\mathrm{QCD}}$ chosen to be a few hundred $\mathrm{MeV}$. The combined effect of the finite spatial volume and potentially missing two-pion tail is estimated using a one-loop finite-volume (FV) lattice-regulated chiral perturbation theory (ChPT) version of Eq. (5.1) of Ref. [32]. Our ChPT computation also agrees with Eq. (2.12) of Ref. [35] after correcting for a missing factor of 2 in the first version of Ref. [35]. The ChPT result is then transformed to position space to obtain $C(t)$. Figure 6 shows a corresponding study of $L_{T}$ for different volumes. We take the difference of $L_{T=20}$ on the $48^{3} \times 96$ lattice used here and

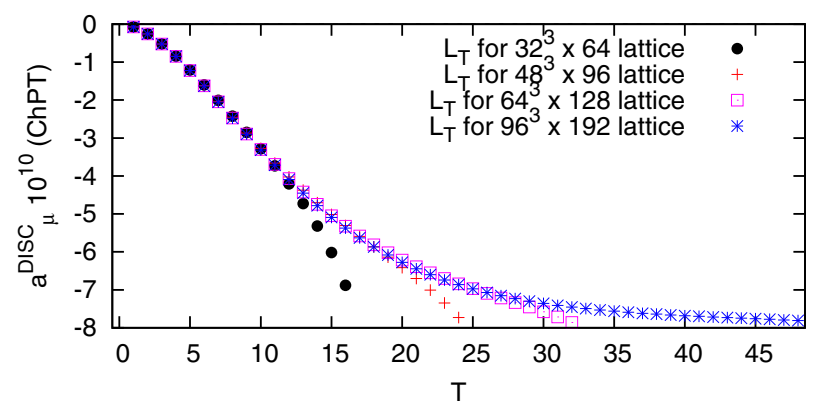

FIG. 6. The leading-order pion-loop contribution in finitevolume ChPT as a function of volume.
$L_{T=48}$ on the $96^{3} \times 192$ lattice and obtain $\delta a_{\mu}{ }^{\mathrm{FV}, \pi \pi}=1.4 \times 10^{-10}$. The remaining long-time effects are estimated by $F_{T=20}$. We compare the result for two fit ranges $F_{T=20}([11, \ldots, 17])=-1.1(6) \times 10^{-10}$ and $F_{T=20}([12, \ldots, 17])=-0.6(0.9) \times 10^{-10}$. We conservatively take the $1 \sigma$ bound $\delta a^{\mathrm{F}_{\mathrm{T}}}=1.7 \times 10^{-10}$ as additional uncertainty. The fit ranges correspond to the largest minimum times before losing a statistical signal, thereby minimizing a potential excited-state contribution in our estimate.

Combining the systematic uncertainties in quadrature, we report our final result,

$$
a_{\mu}^{\mathrm{HVP}(\mathrm{LO}) \mathrm{disc}}=-9.6(3.3)(2.3) \times 10^{-10}
$$

where the first error is statistical and the second systematic.

Before concluding, we note that our result appears to be dominated by very low energy scales. This is not surprising since the signal is expressed explicitly as the difference of light-quark and strange-quark Dirac propagators. We therefore expect energy scales significantly above the strange mass to be suppressed. We already observed this above in the dominance of low modes of the Dirac operator for our signal. Furthermore, our result, which includes the twopion contributions from first principles, is statistically consistent with the one-loop ChPT two-pion contribution of Fig. 6.

Conclusion.-We have presented the first ab initio calculation of the hadronic vacuum polarization disconnected contribution to the muon anomalous magnetic moment at physical pion mass. We were able to obtain our result with modest computational effort utilizing a refined noise-reduction technique explained above. This computation addresses one of the major challenges for a first-principles lattice QCD computation of $a_{\mu}^{\mathrm{HVP}}$ at percent or subpercent precision, necessary to match the anticipated reduction in experimental uncertainty. The uncertainty of the result presented here is already slightly below the current experimental precision and can be reduced further by a straightforward numerical effort.

We thank our RBC and UKQCD collaborators for helpful discussions and support. C.L. is, in particular, indebted to Norman Christ, Masashi Hayakawa, and Chulwoo Jung for helpful comments regarding this manuscript. This calculation was carried out at the Fermilab cluster pi0 as part of the USQCD Collaboration. The eigenvectors were generated under the ALCC Program of the U.S. DOE on the IBM Blue Gene/Q (BG/Q) Mira machine at the Argonne Leadership Class Facility, a U.S. DOE Office of Science facility supported under Award No. De-AC02-06CH11357. T. B. is supported by U.S. DOE Award No. DE-FG02-92ER40716. P. A. B. and A. P. are supported in part by UK STFC Grants No. ST/ M006530/1, No. ST/L000458/1, No. ST/K005790/1, and No. ST/K005804/1 and A. P. is additionally supported by 
Grant No. ST/L000296/1. T. I. and C. L. are supported in part by U.S. DOE Award No. AC-02-98CH10886(BNL). T. I. is supported in part by the Japanese Ministry of Education Grant No. 26400261. L. J. is supported in part by U.S. DOE Award No. de-sc0011941. A. J. is supported by EU Grant No. FP7/2007-2013 ERC Grant No. 279757. K. M. is supported by the National Sciences and Engineering Research Council of Canada. M. S. is supported by the EPSRC Doctoral Training Centre Grant No. EP/G03690X/1.

*Corresponding author. clehner@quark.phy.bnl.gov

[1] D. Hanneke, S. Fogwell, and G. Gabrielse, Phys. Rev. Lett. 100, 120801 (2008).

[2] T. Aoyama, M. Hayakawa, T. Kinoshita, and M. Nio, Phys. Rev. D 91, 033006 (2015).

[3] G. Bennett et al. (Muon G-2), Phys. Rev. D 73, 072003 (2006).

[4] J. Beringer et al. (Particle Data Group), Phys. Rev. D 86, 010001 (2012), including the 2013 update for the 2014 edition at http://pdg.lbl.gov.

[5] J. Grange et al. (Muon g-2), arXiv:1501.06858.

[6] A. Kurz, T. Liu, P. Marquard, and M. Steinhauser, Phys. Lett. B 734, 144 (2014).

[7] M. Davier, A. Hoecker, B. Malaescu, and Z. Zhang, Eur. Phys. J. C 71, 1515 (2011); 72, 1874 (2012).

[8] K. Hagiwara, R. Liao, A. D. Martin, D. Nomura, and T. Teubner, J. Phys. G 38, 085003 (2011).

[9] R. Carey, K. Lynch, J. Miller, B. Roberts, W. Morse et al., The new (G-2) experiment: a proposal to measure the muon anomalous magnetic moment to $+-0.14 \mathrm{ppm}$ precision, FERMILAB-PROPOSAL-0989 (2009).

[10] M. Aoki et al., in Proceedings of PAC2009: Proceedings of the 23rd Particle Accelerator Conference, 2009 (KEK-JPARC, Naka-gun, Ibaraki, Japan, 2009), No. 12.

[11] J. Prades, E. de Rafael, and A. Vainshtein, Adv. Ser. Dir. High Energy Phys. 20, 303 (2009).

[12] T. Blum, N. Christ, M. Hayakawa, T. Izubuchi, L. Jin, and C. Lehner, Phys. Rev. D 93, 014503 (2016).

[13] J. Green, O. Gryniuk, G. von Hippel, H. B. Meyer, and V. Pascalutsa, Phys. Rev. Lett. 115, 222003 (2015).

[14] T. Blum, S. Chowdhury, M. Hayakawa, and T. Izubuchi, Phys. Rev. Lett. 114, 012001 (2015).

[15] V. Gulpers, A. Francis, B. Jager, H. Meyer, G. von Hippel, and H. Wittig, Proc. Sci., LATTICE2014 (2014) 128 [arXiv:1411.7592].

[16] G. S. Bali and G. Endrodi, Phys. Rev. D 92, 054506 (2015).
[17] F. Burger, G. Hotzel, K. Jansen, and M. Petschlies, arXiv:1501.05110.

[18] B. Chakraborty, C. T. H. Davies, J. Koponen, G. P. Lepage, M. J. Peardon, and S. M. Ryan, Phys. Rev. D 93, 074509 (2016).

[19] B. Toth, in the 33rd International Symposium on Lattice Field Theory (2015), http://indico2.riken.jp/indico/getFile.py/ access ? contribId $=266 \&$ session $I d=8 \&$ resId $=0 \&$ material $I d=$ slides\&confId $=1805$.

[20] T. Blum, Phys. Rev. Lett. 91, 052001 (2003); B. E. Lautrup, A. Peterman, and E. de Rafael, Phys. Rep. C3, 193 (1972).

[21] D. Bernecker and H. B. Meyer, Eur. Phys. J A 47, 148 (2011).

[22] X. Feng, S. Hashimoto, G. Hotzel, K. Jansen, M. Petschlies, and D. B. Renner, Phys. Rev. D 88, 034505 (2013).

[23] The appropriate normalization factors of $Z_{V}^{2}$ are of course included in our computation.

[24] J. Foley, K. Jimmy Juge, A. O’Cais, M. Peardon, S. M. Ryan, and J.-I. Skullerud, Comput. Phys. Commun. 172, 145 (2005).

[25] A. Li et al. (xQCD), Phys. Rev. D 82, 114501 (2010).

[26] R. C. Brower, H. Neff, and K. Orginos, in Proceedings, 22nd International Symposium, Lattice 2004, Batavia, USA, June 21-26, 2004 [Nucl. Phys. B, Proc. Suppl. 140, 686 (2005)].

[27] T. Blum et al. (RBC, UKQCD), Phys. Rev. D 93, 074505 (2016).

[28] T. Blum, T. Izubuchi, and E. Shintani, Phys. Rev. D 88, 094503 (2013).

[29] E. Shintani, R. Arthur, T. Blum, T. Izubuchi, C. Jung, and C. Lehner, Phys. Rev. D 91, 114511 (2015).

[30] C. Jung, in the 33rd International Symposium on Lattice Field Theory (2015), http://indico2.riken.jp/indico/getFile.py/ access? contribId=192\&sessionId $=4 \&$ res $I d=0 \&$ materialId $=$ slides\&confId $=1805$.

[31] See Supplemental Material at http://link.aps.org/ supplemental/10.1103/PhysRevLett.116.232002 for additional plots supplementing relevant technical details regarding our results.

[32] M. Della Morte and A. Jüttner, J. High Energy Phys. 11 (2010) 154.

[33] Alternatively taking $T=21$ instead of $T=20$ and repeating our procedure to estimate systematic uncertainties, we find $a_{\mu}^{\mathrm{HVP}(\mathrm{LO}) \text { disc }}=-8.3(4.0)(1.8) \times 10^{-10}$, where the first error is statistical and the second systematic. This value is consistent with our preferred value; however, it has a different balance of statistical and systematic errors.

[34] T. Blum et al. (RBC/UKQCD Collaboration), J. High Energy Phys. 04 (2016) 063.

[35] C. Aubin, T. Blum, P. Chau, M. Golterman, S. Peris, and C. Tu, Phys. Rev. D 93, 054508 (2016). 